\title{
Arsenic content in the Jie River and its release behaviour from river sediments
}

\author{
Guangxiang $\mathrm{Ma}^{1}$, Lizhu Huang ${ }^{1}$, Xingsheng Kang ${ }^{1, *}$, Qing Xu ${ }^{2}$, Guofeng Zhou ${ }^{1}$, Hongkai Fan ${ }^{1}$, Wenhui Zhang ${ }^{1}$, Jianzhang \\ $\mathrm{Sun}^{1}$, Yingjie Meng ${ }^{1}$, Jinwei Qiu ${ }^{1}$, Junjie $\mathrm{Gu}^{1}$, and Tao $\mathrm{Ma}^{1}$
}

${ }^{1}$ Shandong Huankeyuan Environmental Engineering Co., Ltd, Jinan 250013, China

${ }^{2}$ Yishui Branch of Linyi Municipal Ecology and Environment Bureau, Linyi 276000, China

\begin{abstract}
Water and sediment samples of Jie River, China were collected and analyzed. The results showed that the average arsenic volume of water sample was $0.42 \mathrm{mg} \mathrm{L}^{-1}$ present in the Jie River. Among the riverway, the arsenic volume and $\mathrm{pH}$ kept rising with the flow. Standard leaching experiments showed the leachability of the sediments was lower than the hazardous materials permitted threshold volume but the leaching liquid exceeded the environmental quality standard for surface water which means the sediments might be a source to leach arsenic into aqueous system. In order to figure out the actual situation, simulated leaching experiments were performed. The results revealed that the sediments leaching could aggravate the water quality which was mostly influenced by the agitation and temperature. Solidification procedure was tested and suggested that with clean sand paved on the sediment, the amount of leaching arsenic will decrease $77.89 \%$. Thus, it is of great help to apply cleaned sediments to remediate the contaminated sediments to reduce the leaching of arsenic into the aqueous system.
\end{abstract}

\section{Introduction}

Arsenic accumulation in river sediments has received great concern due to its high toxicity. As an enormous recipient, river sediments receive micro pollutants, trace metallic, and metalloid elements like arsenic ${ }^{[1]}$. Many arsenic polluted rivers have been found because of the human activities, and many people suffered from the arsenic-contaminated water ${ }^{[2]}$. In general, the arsenic is ubiquitous in the rivers around the word ${ }^{[3]}$, the average content of arsenic in river sediments is $5 \mathrm{mg} \mathrm{kg}^{-1}$. In some severe contaminated area such as mining area, the arsenic content in river sediments exceeds $200 \mathrm{mg} \mathrm{kg}^{-1}$. High concentration of arsenic in sediments poses great health threat to the human beings because the arsenic in sediment might leach into the aqueous system through desorption resulting in arsenic accumulation in aquatic life $\mathrm{e}^{[4]}$. The arsenic will influence human health through the food chain eventually ${ }^{[5]}$. Therefore, it is critical to estimate the risk of the arsenic occurrence amount in sediments to protect aquatic life and human health.

In order to estimate the risk of the arsenic in rivers, the total content arsenic in aqueous system and river sediments is needed ${ }^{[6]}$. However, only total content cannot characteristic the leaching behaviour of the sediments due to different chemical components ${ }^{[7]}$. Therefore, the leaching behaviour of sediments is also essential to evaluate its risk assessment and subsequently treat the arsenic-contaminated sediments.

Jie River, which is located in Zhaoyuan (Shandong province), is the biggest river of the city. Zhaoyuan is known for abundant gold reserves, has discharged numerous mining wastewater into the Jie River causing severe pollution. Recently, the local government has paid more attention to the river pollution issue, it was found that the arsenic is the most troublesome pollutant in the Jie River. Therefore, we investigated the arsenic content in Jie River and its release from the river sediments. The main objectives of this research were to (1) to determine the arsenic pollution situation in the aqueous system and river sediments in Jie River; (2) to verify the leachability of the river sediments in Jie River; and (3) to offer effective action to alleviate the arsenic pollution problem.

\section{Materials and methods}

\subsection{Water and sediments sampling}

The water and the sediments were collected according to Fig. 1, the black label mean water sampling sites and the blue label mean the sediments sampling sites. The $10^{\text {th }}$ water sampling site is the downstream of the river. In general, 10 surface water samples and 8 sediments samples were collected. To minimize random error, the water samples were collected at four days and three different samples were collected at each sampling site. All water samples were acidized to ensure the $\mathrm{pH}$ below 2 using sulfuric acid. For sediments sampling, three different sampling layers were applied as below: first layer (0-20 cm depth from surface); second layer (20-40

\footnotetext{
* Corresponding author:
} 
$\mathrm{cm}$ depth from surface); and third layer (40-70 $\mathrm{cm}$ depth from surface). Like the water samples, sediments were collected triplicated at each sediment sampling position.

\subsection{Batch experiments}

Natural release experiments were conducted as follow: 4:1 deionized water: sediments $(\mathrm{v} / \mathrm{v})$ were placed in 1000 $\mathrm{ml}$ beaker which was enclosure by tinfoil for the prevent of the light. $50 \mathrm{ml}$ water samples were collected at 12, 24, $36,48,60,72,84$, and $96 \mathrm{~h}$. After each collecting, $50 \mathrm{ml}$ deionized water was added into the beaker to maintain the total aqueous volume. The $\mathrm{pH}$ effect was studied by adding $0.1 \mathrm{M} \mathrm{HNO}_{3}$ and $0.1 \mathrm{M} \mathrm{NaOH}$ to maintain different $\mathrm{pH}(6,7,8,8.5,9$, and 9.5). Stirring effect was performed by using $60 \mathrm{rpm}$ speeds to investigate the relationship between agitation and arsenic leaching behaviour. The effect of temperature was simulated by temperature incubator at three different temperatures $(5$, 15 , and $25^{\circ} \mathrm{C}$ ).

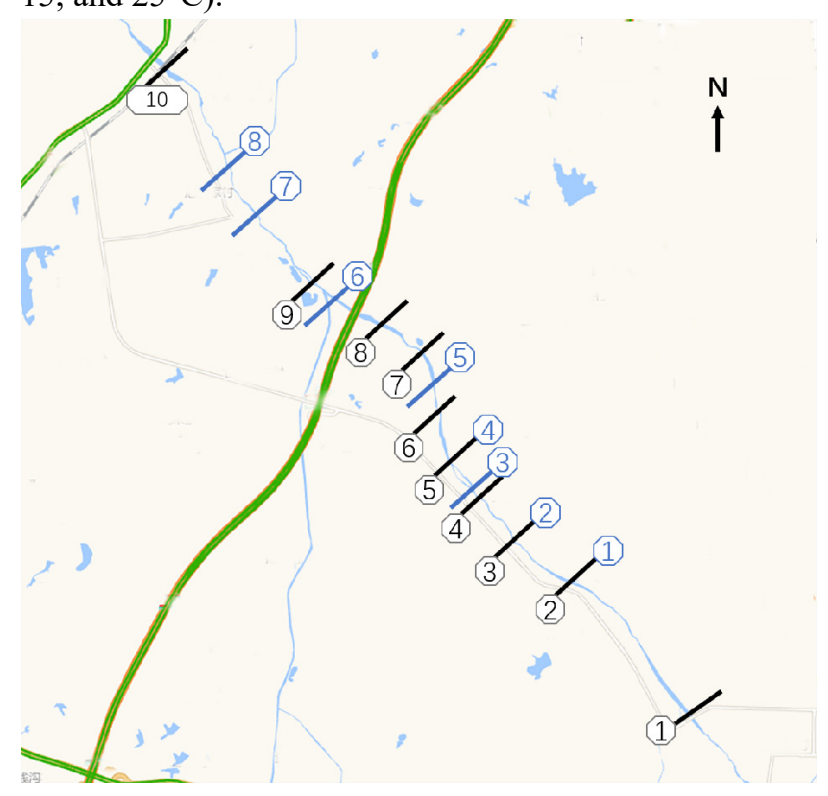

Fig. 1. Schematic graph of the sampling sites.

\subsection{Analytical methods}

The arsenic content of water was filtrated by $0.45 \mu \mathrm{m}$ filter and analysed by atomic fluorescence spectroscopy (AFS). The sediments leaching fluids were acid digestion first with a solution of $6: 3: 2 \mathrm{HNO}_{3}: \mathrm{H}_{2} \mathrm{O}_{2}: \mathrm{HF}$ $(\mathrm{v} / \mathrm{v})$, then the analyse of arsenic content in the digestion liquids was conducted as mentioned. The toxicity leaching experiments were followed by Solid-waste Extraction Procedure for Leaching Toxicity-Sulphuric Acid \& Nitric Acid Method (HJ/T299-2007). The pH of water samples was analysed by HACH HQ14D $\mathrm{pH}$ meter. The statistical analysis was based on $t$ test and one-way analysis of variance (ANOVA) in order to test the statistically significant differences. All statistical tests were carried out using the Graph Pad Prism 8.3.0 software package. Triplicate experiments were carried out for all samples and the value in this study was average.

\section{Results and discussion}

\subsection{Arsenic content and pH of surface water}

As shown in Fig. 2 and Fig. 3, the pH value and arsenic concentration kept rising with river flowing downstream, the highest $\mathrm{pH} 8.97$ was observed at $10^{\text {th }}$ sampling site and the highest arsenic concentration $0.082 \mathrm{mg} \mathrm{L}^{-1}$ was observed at $8^{\text {th }}$ sampling site. The $\mathrm{pH}$ of $10^{\text {th }}$ sampling water was $11.01 \%$ higher than $1^{\text {st }}(\mathrm{p}<0.0001)$ which can be explained by the rapid increase of arsenic $(p<0.0001)$. The arsenic concentrations of $6^{\text {th }}, 7^{\text {th }}, 8^{\text {th }}, 9^{\text {th }}$, and $10^{\text {th }}$ sampling site exceeded the permitted threshold value (0.05 $\left.\mathrm{mg} \mathrm{L}^{-1}\right)$ and had maintained the high level continuously. This trend, hypothetically not caused by illegal industrial discharge, will only be the consequence of the arsenic release from sediments.

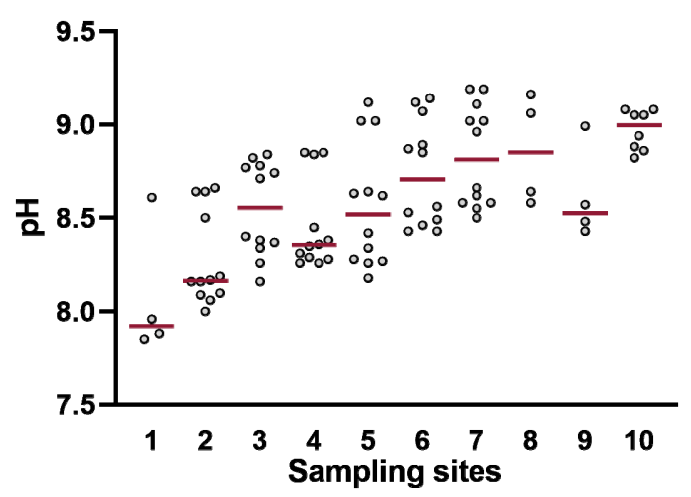

Fig. 2. $\mathrm{pH}$ values at different sampling site.

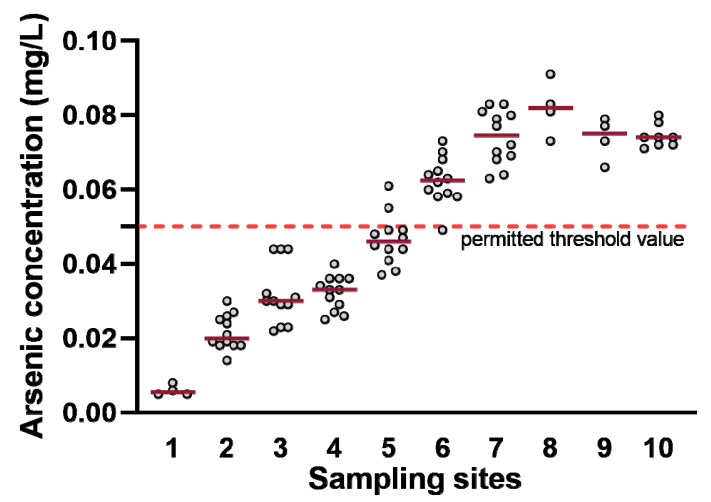

Fig. 3. Arsenic concentrations at different sampling site.

\subsection{Arsenic leaching behaviour}

\subsubsection{Standard leaching experiment}

The standard leaching experiments were conducted followed by HJ/T299-2007. The leaching experiments were conducted using sediments from different sites with different depths. It can be seen from Fig. 4 that the leaching concentration of arsenic increased as sampling depths became deeper especially at third layer. The average leaching concentration of third layer is $48.70 \%$ and $41.32 \%$ higher than first layer and second layer, respectively. It is demonstrated that the depth of sediments is positively related to the arsenic content. 
With higher arsenic content, arsenic present in sediments might leach into water more easily.

Fig. 5 shows that the distribution of arsenic was irregular, sediments in different sites had different leaching concentration to the water. Although all of them did not exceed the hazardous materials threshold volume (GB5085.3-2007, $5 \mathrm{mg} \mathrm{L}^{-1}$ ), considering the sediments volume, it is possible for sediments to leach large amount of arsenic into aqueous system. In order to figure out the leaching behaviors of sediments under natural conditions, several natural leaching experiments were carried out.

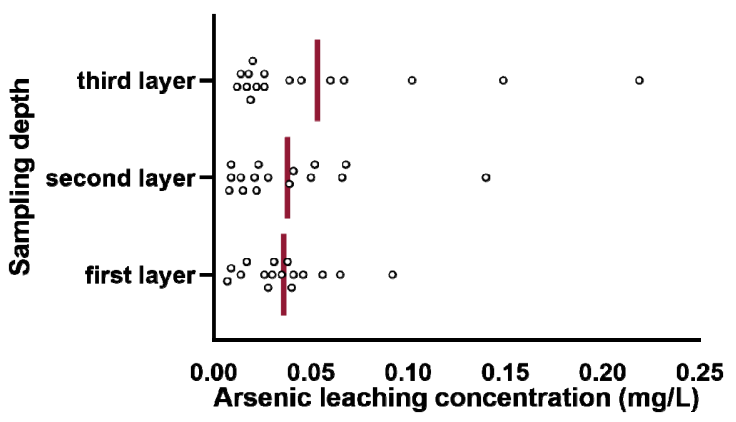

Fig. 4. Arsenic leaching concentrations from sediments at different sampling depths.

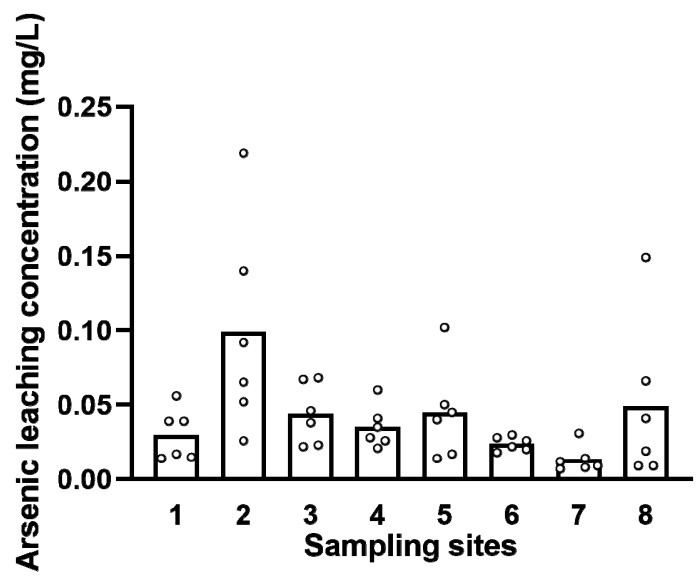

Fig. 5. Arsenic leaching concentrations from sediments at different sampling sites.

\subsubsection{Simulated leaching experiment}

Standard toxicity leaching can be used to determine the extreme leaching behaviour of waste, nevertheless, the natural leaching behaviour of sediments was more significant to be revealed because it was designed to simulate the sediments leaching behaviour. In this study, static experiment, orthogonal experiment, and solidification experiment were conducted ${ }^{[8]}$.

The result of static leaching experiments was shown in Fig. 6, the highest leaching concentration was lower than $15 \mu \mathrm{g} \mathrm{\textrm {L } ^ { - 1 }}$, revealed the unordered leaching behaviour. With leaching duration rose, most of the leaching concentration increased followed by a decline (sampling site 2, 3, 5,6, and 8), it demonstrated that the leached arsenic would settle down onto the sediments and be absorbed again. However, in the long term, the leached arsenic will increase and inevitably accumulate in the aqueous system ${ }^{[9]}$.

The orthogonal test was based on three factors: temperature, $\mathrm{pH}$, and agitation. The orthogonal test scheme and results analysis are presented in Table 1 . The different range values were $R_{\text {temperature }}=6.722667$, $\mathrm{R}_{\mathrm{pH}}=0.931333$, and $\mathrm{R}_{\mathrm{agitation}}=10.503$, the order of impact of these three factors was: agitation $>$ temperature $>\mathrm{pH}$. This result demonstrated that the arsenic leached into the river was mostly caused by agitation and temperature, the shear force of the river mixed up the sediments and make it easier for the arsenic to leach into the water. The $\mathrm{pH}$ value of water changed the leaching concentration barely, unlike the other researches clarified ${ }^{[10]}$, might caused by the different properties of sediments.
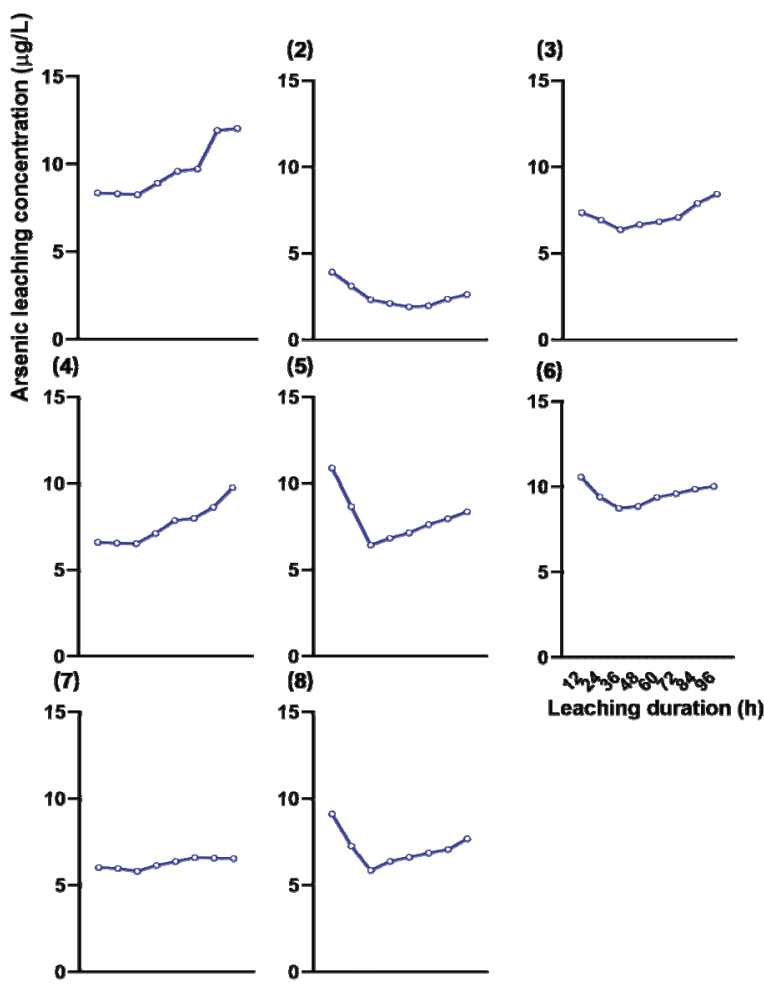

Fig. 6. Static leaching behaviour of sediments at different sampling sites.

\subsubsection{Solidification experiment}

In order to provide remediation solution for the nonpoint kind pollution type like Jie River and to develop polluted river sediments management strategies, better solidification method was demanded. In this study, the contaminated sediments were washed and backfill the river channel at the surface of the sediments. In general, 2:1 cleaned sediment: contaminated sediment $(\mathrm{v} / \mathrm{v})$ was introduced to $1000 \mathrm{ml}$ beaker with $800 \mathrm{ml}$ deionized water.

The leaching concentration was tested at different time with different $\mathrm{pH}$ (Fig. 7). The leaching concentration varied at a smaller section compared to nosolidification leaching experiments. All samples stayed stable from 12 to 96 hours, this means the solidification was functional. The maximum leaching volume was 
$3.598 \mu \mathrm{g} \mathrm{L}^{-1}$ at $\mathrm{pH}=9$, comparing with previous, has lowered $77.89 \%$. Significant descend was noted in comparison between solidification experiment and control group (Fig. 8).

The results showed that the leaching concentration decreased pronouncedly using solidification. For solidification group and control group, there were statistically significant for the leaching of arsenic at $\mathrm{pH}=8.0,8.5,9.0$, and $9.5(\mathrm{p}<0.0001)$. The cleaned sediments paved on the contaminated sediments made the arsenic present in deeper sediments more difficult to leach into the water, meanwhile, the cleaned sediments can be a source to adsorb some arsenic, reducing the amount of arsenic transferred into water.

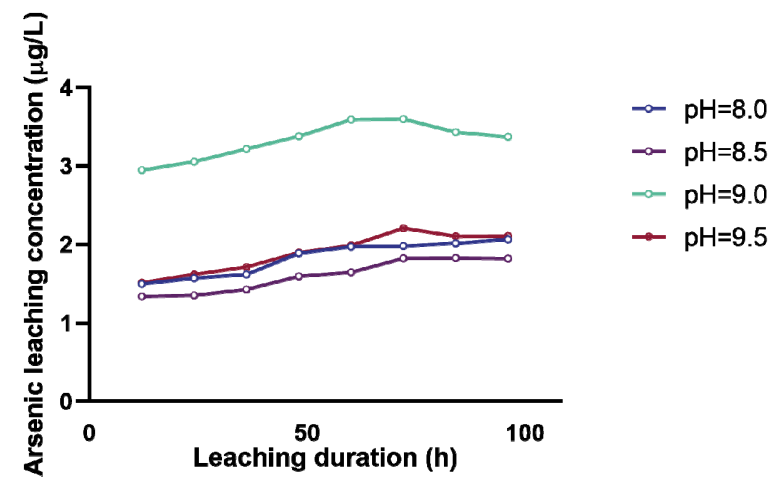

Fig. 7. Leaching behaviour after solidification procedure.

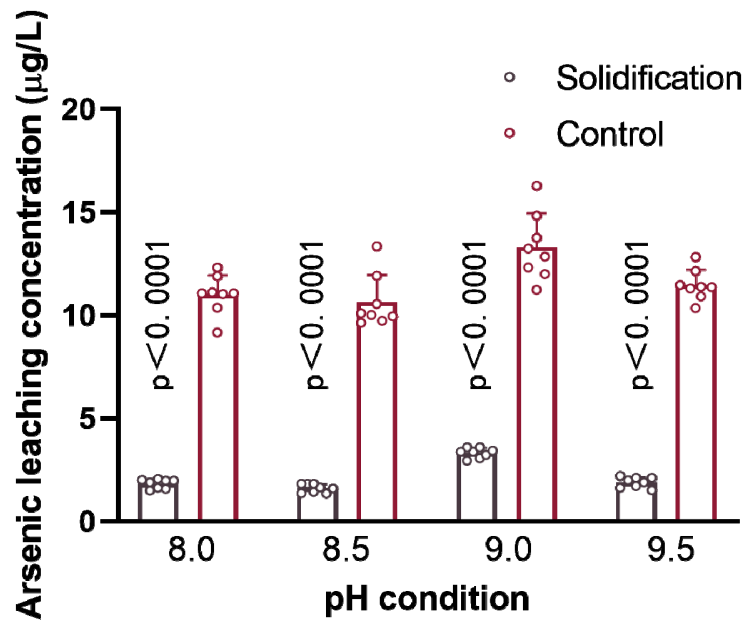

Fig. 8. Arsenic leaching comparison between solidification experiment and control group.

Table. 1. Orthogonal test and leaching values.

Factors

\begin{tabular}{ccccc}
\cline { 2 - 5 } Number & $\begin{array}{c}\text { Temperat } \\
\text { ure }\left({ }^{\circ} \mathrm{C}\right)\end{array}$ & $\mathrm{pH}$ & $\begin{array}{c}\text { Agitati } \\
\text { on } \\
(\mathrm{RPM})\end{array}$ & $\begin{array}{c}\text { Leaching } \\
\text { concentrati } \\
\text { on }\left(\mu \mathrm{g} \mathrm{L} \mathrm{L}^{-1}\right)\end{array}$ \\
\cline { 2 - 5 } 1 & 5 & 7 & 0 & 4.580 \\
2 & 5 & 9 & 60 & 9.859 \\
3 & 5 & 10 & 120 & 13.861 \\
4 & 15 & 7 & 60 & 11.214 \\
\hline
\end{tabular}

\begin{tabular}{ccccc}
\hline 5 & 15 & 9 & 120 & 16.685 \\
6 & 15 & 10 & 0 & 4.360 \\
7 & 25 & 7 & 120 & 20.915 \\
8 & 25 & 9 & 0 & 11.012 \\
9 & 25 & 10 & 60 & 16.541 \\
$\mathrm{~K}_{1}$ & 28.300 & 36.709 & 19.952 & \\
& & & & \\
$\mathrm{~K}_{2}$ & 32.259 & 37.556 & 37.614 & \\
& & & & \\
$\mathrm{~K}_{3}$ & 48.468 & 34.762 & 51.461 & \\
& & & & \\
$\mathrm{R}$ & 6.722667 & 0.9313 & 10.503 & \\
\hline
\end{tabular}

\section{Conclusion}

The total arsenic content in Jie River ranged from 0.05 to $0.091 \mathrm{mg} \mathrm{L}^{-1}$, with an average of $0.048 \mathrm{mg} \mathrm{L}^{-1}$. The leaching arsenic from sediments in Jie River ranged from $0.007 \mathrm{mg} \mathrm{L}^{-1}$ to $0.219 \mathrm{mg} \mathrm{L}^{-1}$, with an average of 0.042 $\mathrm{mg} \mathrm{L}^{-1}$. Most of the samples were not significantly contaminated by arsenic, but simulated leaching experiment raised unsafe concerns. In this study, cleaned sediments were recommended to apply for the remediation of the leachable contaminated sediments and the experiments data showed great promising prospects. In general, the contaminated sediments need to be remediated to decrease the leachability of the arsenic and lower the health risk.

\section{Acknowledgment}

We thank the Foundation of contaminated sediment remediation in Shandong Province (No. 2017-HCZBLY003) and Shandong Key Scientific and Technical Innovation Project (No. 2018YFJH0902) for supporting this research.

\section{References}

1 Gottfried TW Wittmann, Metal pollution in the aquatic environment. (Springer-Verlag, 1981).

2 B. Kumar Mandal, K. T. Suzuki, Talanta 58 (1), 201-235 (2002).

3 A. Sarkar, B. Paul, Chemosphere 158, 37-49 (2016).

4 P. L. Smedley, H. B. Nicolli, D. M. J. Macdonald, A. J. Barros, and J. O. Tullio, APPL GEOCHEM 17 (3), 259-284 (2002).

5 J. Bundschuh, B. Nath, P. Bhattacharya, C. Liu, M. Aurora Armienta, M. V. M. López, D. L. Lopez, Jiin-Shuh Jean, L. Cornejo, L. Fagundes Lauer 
Macedo, and A. Tenuta Filho, SCI TOTAL ENVIRON 429, 92-106 (2012).

6 M. Berg, P. Thi Kim Trang, C. Stengel, J. Buschmann, P. Hung Viet, N. Van Dan, W. Giger, and D. Stüben, CHEM GEOL 249 (1), 91-112 (2008).

7 F. M. G. Tack and M. G. Verloo, Int J Environ Anal Chem 59 (2-4), 225-238 (1995).

8 V. Cappuyns and R. Swennen, J. Hazard. Mater. 158 (1), 185-195 (2008).

9 D. Sánchez-Rodas, J. Luis Gómez-Ariza, I. Giráldez, A. Velasco, and E. Morales, SCI TOTAL ENVIRON 345 (1), 207-217 (2005).

10 P. H. Masscheleyn, R. D. Delaune, and W. H. Patrick, EST. 25 (8), 1414-1419 (1991). 